\title{
Chronic Administration of Curcuma longa Extract Improves Spatial Memory-Related Learning Ability in Aged Rats by Inhibiting Brain Cortico-Hippocampal Oxidative Stress and TNF $\alpha$
}

\author{
Fowzia Akter, Mozammel Haque, Jahirul Islam, Asiqur Rahaman, Sujan Bhowmick, \\ Shahdat Hossain* \\ Laboratory of Alternative Medicine \& Behavioral Neurosciences, Department of Biochemistry and Molecular \\ Biology, Jahangirnagar University, Savar, Dhaka, Bangladesh \\ Email: fowziaselina@gmail.com, adieb_bio.ju37@yahoo.com, jahir1039@gmail.com, rafibioju@gmail.com, \\ sujan.bmbju@gmail.com, shahdat@dhaka.net
}

Received 31 May 2015; accepted 28 August 2015; published 1 September 2015

Copyright (C) 2015 by authors and Scientific Research Publishing Inc.

This work is licensed under the Creative Commons Attribution International License (CC BY).

http://creativecommons.org/licenses/by/4.0/

(c) (i) Open Access

\section{Abstract}

We studied on the effect of Curcuma longa extract on spatial learning-related memory ability of old rats in eight-arm radial maze task. Rats were randomly divided into two groups: one group was orally administered $100 \mathrm{mg} / \mathrm{KgBW} /$ day $C$. longa extract (CLE) dissolved in deionized water and the other group was administered the vehicle alone for 10 weeks. The rats were tested with the partially baited eight-arm radial maze to evaluate two types of spatial memory-related learning ability displayed by reference memory errors (RMEs) and working memory errors (WMEs). Chronic administration of CLE significantly decreased the number of RMEs and WMEs, concurrently with the decreases in the cortico-hippocampal levels of lipid peroxides (LPO) and tumor necrosis factor alpha (TNF- $\alpha$ ). In a parallel set of experiments, CLE-pretreated rats of the same age group were subjected to hypoxia-reperfusion injury by carotid artery occlusion to induce oxidative stress in the brains in order to examine whether such an in vivo hypoxia-induced oxidative stress could be ameliorated by the extract. Again, the levels of LPO were significantly decreased in the cortico-hippocampal tissues of the CLE-fed hypoxic rats. The histology of the brains also revealed that the CLE-pretreated rats had retained improved cellular integrity. Finally, our results provide the evidence that oral administration of $C$. longa extract increases the defense against oxidative stress and proinflammatory TNF- $\alpha$, concurrently with the improvement of memory-re-

\footnotetext{
"Corresponding author.
}

How to cite this paper: Akter, F., Haque, M., Islam, J., Rahaman, A., Bhowmick, S. and Hossain, S. (2015) Chronic Administration of Curcuma longa Extract Improves Spatial Memory-Related Learning Ability in Aged Rats by Inhibiting Brain Cortico-Hippocampal Oxidative Stress and TNFa. Advances in Alzheimer's Disease, 4, 78-89.

http://dx.doi.org/10.4236/aad.2015.43008 
lated brain cognitive ability of the aged rats.

\title{
Keywords
}

\author{
Curcuma longa, Spatial Memory, Eight-Arm Radial Maze, Carotid Artery Occlusion, TNF $\alpha$, Lipid \\ Peroxidation, Hypoxia
}

\section{Introduction}

Curcuma longa, commonly known as turmeric, is one of the most studied medicinal plants with potential anticancer, anti-inflammatory and neuroprotective effects [1] [2]. The most important active constituent of $C$. longa is the curcumin, which is responsible for the vibrant yellow color of the turmeric [3] [4]. The yellow pigment curcumin (diferuloylmethane) is a low molecular weight polyphenol which is mostly implicated in the antioxidant and anti-inflammatory effects [5]. Studies have shown that curcumin prevents lipid peroxidation in animal brains [6], inhibits the formation of beta-amyloid oligomers and fibrils both in vitro and in vivo [7]-[10], and attenuates inflammatory response of brain microglial cells [11] [12]. One study has reported that curcumin is more potent than vitamin E as a free radical scavenger [13]. Recently Pyrzanowska et al. (2010) reported that the extract of $C$. longa enhances the learning and memory of old rats in the water maze [14]. Our aim in this study was to examine the hypothesis that curcumin might play an important role in spatial memory formation. Although various studies reported positive effects of curcumin on the spatial learning ability, in most of the cases Morris water maze paradigm was used to evaluate the learning ability. It is, however, difficult to interpret the learning abilities measured in one procedure due to drug- or lesion-induced deficits [15]. Therefore, we examined the effect of chronic administration of $C$. longa extract (CLE) on the spatial memory by eight-arm radial maze, and whether CLE administration concurrently affects the levels of lipid peroxidation and tumor necrosis factor alpha (TNF $\alpha$ ) in the cortex/hippocampus regions of old rat brains. Radial maze measures learning ability by measuring two types of memory, reference memory and working memory. Reference memory is the information that should be retained until the next trial. Working memory is the information that disappears in a short time. In the present experimental paradigm, "entries into unbaited arms" and "repeated entries" into visited arms were defined as reference memory errors and working memory errors, respectively. Thus the effects of $C$. longa extract on the reference memory errors and working memory errors were evaluated. Besides, forebrain ischemia was induced by temporary occlusion of common carotid artery to examine whether chronic oral preadministration of C. longa extract prevents against the oxidative stress in the brains of the hypoxic rats. Finally, the outcomes of the effect of $C$. longa extract on hypoxia-induced oxidative stress were correlated with the effect of $C$. longa extract on spatial memory of old rats.

\section{Materials and Methods}

\subsection{Animals}

Male Long Evans rats (75-week-old) taken from the University Animal House, were housed in a room under controlled temperature $\left(25^{\circ} \mathrm{C} \pm 2^{\circ} \mathrm{C}\right)$, relative humidity (50\% $\left.\pm 10 \%\right)$ and light-dark cycles (light: 06:00 - 18:00; dark: 18:00 - 06:00). They were fed a standard pellet diet and water $a b$ libitum. Two parallel independent experiments were run: the effects of oral administration of $C$. longa extract (CLE) on the learning-related spatial memory of old rats (Behavioral experiment), and oxidative stress and histology of the hippocampus of the old rats subjected to hypoxia-reperfusion injury. For behavioral experiment, 21 rats were randomly divided into two groups: the Control (vehicle) group $(\mathrm{n}=11)$ and the $C$. longa extract (CLE)-fed group $(\mathrm{n}=10)$. The $C$. longa extract was suspended in water and fed orally by gastric tube at $100 \mathrm{mg} / \mathrm{kg} \mathrm{BW} /$ day; the rats of the Control group were orally fed a similar volume of water only. Oral administration of the CLE was continued until the completion of behavioral experiments. For hypoxia-reperfusion injury model (carotid artery occlusion experiment), 20 rats were randomly divided into four groups each consisting of 5 rats: Control (vehicle) group; $C$. longa Extract (CLE)-fed group; Hypoxia-alone group and Hypoxia + CLE-fed group. The C. longa extract was suspended in water and fed orally by gastric tube at $100 \mathrm{mg} / \mathrm{kg}$ BW/day; the rats of the Control and/or Hypoxia- 
alone group were orally fed a similar volume of water. Administration of the CLE was continued for six weeks before surgery.

All the rats were cared for and killed in accordance with the procedures outlined in the Guidelines for Animal Experimentation of the University, compiled from the Guidelines for Animal Experimentation of the Bangladesh Association for Laboratory Animal Science.

\subsection{Behavioral Experiment}

A standard eight-arm radial maze was used for the estimation of learning-related memory ability, as previously described [16]. The maze was placed in a closed room with a number of visual cues: ceiling lights, curtained door and windows and some fixed photographic signs. The experimenter maintained a constant position beside the maze and observed the behavior of the rats. In the experimental paradigm, the animal received food rewards at specific points along the path of the track of the eight-arm radial maze [17]. Three weeks after starting the CLE administration, each rat was placed on a limited-food schedule designed to maintain the weight at $80 \%$ - 85\% of the free-feeding weight and was handled 5 min daily for five consecutive days to familiarize with the experimenter. Then, the rats were familiarized with the apparatus for five days, where $45 \mathrm{mg}$ of reward pellets was scattered throughout the maze.

During the testing period, each rat was given one daily trial for seven days/week. A trial consisted of baiting only four arms with reward pellet and placing the rat in the center platform facing a randomly selected arm. On that occasion, the same four arms were baited consistently for any one animal, which were determined by the raffle system. The testing continued for seven weeks and involved two parameters of memory-related functions: Reference Memory Errors (RMEs), entry into unbaited arms; and Working Memory Errors (WME), repeated entry into arms that had already been visited within a trial.

\subsection{Preparation of Brain Tissues}

After completion of the eight-arm maze task, the rats were anesthetized with ketamine $(100 \mathrm{mg} / \mathrm{kg}$ body weight, i.p.) to collect blood, brains and other tissues. The brains were rapidly removed and dissected into cerebral cortex and hippocampus, as described previously [16]. Then brain tissues were immediately homogenized in ice-cold phosphate buffer (pH 7.4), containing $1 \mathrm{mM}$ PMSF using Dounce tissue homogenizer. The homogenates were immediately subjected to the biochemical assays and/or stored at $-80^{\circ} \mathrm{C}$.

\subsection{Lipid Peroxidation (LPO) Assay}

The levels of lipid peroxide (LPO) in the brain tissues were assessed by the thiobarbituric acid-reactive substances assay of Ohkawa et al. (1979) [18], as previously described [19]. The reaction mixture, containing 0.1 $\mathrm{ml}$ homogenate, $0.1 \mathrm{ml} 8.1 \%$ sodium dodecyl sulfate and $2.0 \mathrm{ml}$ of a $0.8 \%$ solution of thiobarbituric acid in $20 \%$ acetic acid solution (pH 3.5), was made up to a final volume of $3.0 \mathrm{ml}$ with distilled water. The mixture was heated at $95^{\circ} \mathrm{C}$ for $60 \mathrm{~min}$. After cooling with tap water, $2.0 \mathrm{ml}$ of a mixture of n-butanol and pyridine $(15: 1, \mathrm{v} / \mathrm{v})$ was added, and the whole mixture was shaken vigorously for $10 \mathrm{~min}$. After centrifugation at $1000 \times \mathrm{g}$ for $10 \mathrm{~min}$, the absorbance of the organic (upper) layer was measured at $532 \mathrm{~nm}$. The data are expressed as nmol of malondialdehyde/mg protein using 1,1,3,3-tetraethoxypropane as a standard.

Total protein was estimated by the method of Lowry et al. (1951) [20].

\subsection{Determination of TNF- $\alpha$ Protein Levels in the Cortico-Hippocampal Tissues}

The levels of TNF- $\alpha$ in the cortex/hippocampus were estimated according to the previously described method [21]. Briefly, the multi-well plate was coated with cytosolic fraction of cortex/hippocampus in $0.1 \mathrm{M}$ sodium bicarbonate, $\mathrm{pH} 9.6$ at $4^{\circ} \mathrm{C}$ overnight and then blocked with $1 \%$ BSA in Tris-buffered saline (TBS). The primary antibody anti-rabbit TNF- $\alpha$ (Santa Cruz Biotechnology, CA, USA), at 1:1000 dilutions was incubated in the plate for overnight at $4^{\circ} \mathrm{C}$. Horseradish peroxidase-coupled anti-rabbit IgG (Biosource International, Inc., Camarillo, CA, USA) was used as the secondary antibody and incubated further for $2 \mathrm{~h}$ at room temperature before the addition of tetramethylbenzidine substrate to develop color. The reaction was stopped by addition of $0.1 \mathrm{~N} \mathrm{HCl}$ after incubation for $30 \mathrm{~min}$ at room temperature. Wells coated with only $0.1 \mathrm{M}$ carbonate buffer, $\mathrm{pH} 9.6$ were used as blank. The plates were analyzed with a multiwell plate reader (ErbaLisascan II, Mannheim, Germany) at $450 \mathrm{~nm}$. 


\subsection{Hypoxia Experiment: Surgery for Unilateral Common Carotid Artery Occlusion}

The rats were subjected to occlusion of left common carotid artery (LCCA) [22] [23]. After anesthesia by ketamine $(100 \mathrm{mg} / \mathrm{kg}$ body weight, i.p.), skin was carefully incised in the left-side of neck to expose the LCCA. Ischemia-reperfusion injury was produced by blocking LCCA for 45 minutes with the aid of metallic clips. Afterwards, the clips were released for reperfusion for 30 minutes. Body temperature was maintained at about $37^{\circ} \mathrm{C}$ during the whole period with the help of a heating pad. Then the hypoxic rats were killed, their hippocampus and cerebral cortex were separated on ice and homogenized [16] and subjected to lipid peroxidation (LPO) assay (as described above). Brains of some animals were fixed in 10\% neutral buffered formalin for histology.

\subsection{Histology}

Formalin fixed brains were processed with paraffin. Sections with a thickness of $10 \mu \mathrm{m}$ were cut and stained with hematoxylin and eosin for examining the overall morphology. Sections were mounted on gelatin-chromealum coated slides. The microscopic observation was made by fluorescent microscope normal spectra in $100 \times$ and $10 \times$.

\subsection{In Vitro Antioxidative Properties of C. longa}

Total phenolic content of $C$. longa extract was determined by using the Folin-Ciocalteu's method, as previously described [24] [25]. Polyphenol compounds present in the extract reduce phosphotungustomolybdic acid of Folin-Ciocalteu's reagent in alkaline solution to produce a highly blue colored solution. The color complex was measured at $765 \mathrm{~nm}$. The concentration of total phenol compounds in the extract was determined against gallic acid as standard compound (mg of gallic acid equivalent/gm of extract). Total flavonoids (TF) were measured following a previously reported spectrophotometric method [26] against quercetin as a reference standard (mg of quercetin equivalent/gm of extract). The DPPH (2, 2 diphenyl-1-picrylhydrazyl) free radical scavenging activity of $C$. longa extract was evaluated as described previously [27]. The free radical scavenging ability was evaluated through recording the change of absorbance produced by the reduction of the absorbance of $0.2 \mathrm{mM}$ of DPPH. DPPH scavenging potential was calculated as (\%):

$$
\frac{\left[\mathrm{A}_{\mathrm{DPPH}}\right]-\left[\mathrm{A}_{\mathrm{DPPH}+\mathrm{Scavenger}}\right]}{\left[\mathrm{A}_{\mathrm{DPPH}}\right]} \times 100
$$

where, $A_{\mathrm{DPPH}}=$ Absorbance of the DPPH (0.2 mM) alone; $A_{\mathrm{DPPH}+\text { Scavenger }}=$ Absorbance of the DPPH in the presence of scavenger (here, curcumin standard and/or $C$. longa extract).

The in vitro anti-LPO activity of $C$. longa extract was monitored by directly inducing oxidative stress (OS) in the brain tissue homogenates by Fenton's reagent $\left(100 \mu \mathrm{l}\right.$ of $\left.\mathrm{H}_{2} \mathrm{O}_{2} 45 \mathrm{mM}: \mathrm{FeSO}_{4} 2 \mathrm{mM}\right)$. Briefly, the samples, homogeante $(0.1 \mathrm{ml})$ - alone (control); homogenate + Fenton's reagent (OS); homogenate + OS $+100 \mu \mathrm{l} C$. longa extract $(10 \mathrm{mg} / \mathrm{ml})\left(\mathrm{OS}+\mathrm{C}\right.$. longa) were incubated at $37^{\circ} \mathrm{C}$ for $2 \mathrm{~h}$. Afterwards, the levels of LPO in the samples were measured, as indicator of oxidative stress.

\subsection{Statistical Analysis}

All results are expressed as mean \pm standard error of the mean (SEM). The statistical significance of the differences in the behavioral scores between the control and the $C$. longa extract group was determined by two-way ANOVA, with group and block number as factors. One-way ANOVA was used for the analyses of in vitro anti-LPO activity of the extract and those of the LPO levels of the hypoxic rats. The statistical significance of the differences in the mean values of the levels of LPO and TNF- $\alpha$ protein between the groups were determined by student's $t$-test. Software's used were StatView ${ }^{\circledR} 4.01$ (MindVision Software, Abacus Concepts, Inc., Berkeley, CA, USA), GRAPHPAD PRISM ${ }^{\circledR}$ (version 5.00; GraphPad Software Inc., San Diego, CA, USA). A value of P $<0.05$ was considered as significant.

\section{Results}

\subsection{Body Weight and Food Intake of the Rats after Maze Tests}

There was a gradual and significant increase in body weight gain of both control and CLE-fed rats during the 
experimental period. However, the differences in body weight gain between the control and C. longa extract-fed rats were not statistically significant seen (data not shown). Food intake between the control and experimental groups were not also different. No mortality was observed in either of the rat groups.

\subsection{Effect of Oral Administration of $C$. longa Extract on the Radial Arm Maze Task}

Spatial memory is highly relevant in biology because it is related with both individual and species survival. Among behavioral tests, one of the most suitable devices for measuring spatial learning and memory is the radial maze. Thus we used eight-arm radial maze paradigm for the determination of the effect of oral administration of $C$. longa extract on the spatial memory of old rats in our investigation. During the pre-training procedure the data collection showed that rats required the maximum amount of time (300 seconds) in order to eat all the baits. Thus data acquisition was completed within 300 seconds. Figure 1(A) shows the effect of chronic administration of $C$. longa extract on reference memory-related learning ability in aged rats. The score is expressed as mean number of RMEs for each group, with data averaged over blocks of six trials. The analysis revealed a significant main effect of blocks of trials $(\mathrm{F}=307.0 ; \mathrm{P}<0.0001)$ and group $(\mathrm{F}=22.85 ; \mathrm{P}<0.0001)$ on the number of RMEs. The analysis also revealed a significant block and groups (Control and $C$. longa extract-fed group) interactions ( $\mathrm{F}=19.34$; $\mathrm{P}<0.0001)$. Figure $1(\mathrm{~B})$ shows the effect of chronic administration of $C$. longa extract on working memory-related learning ability. The score is expressed as the mean number of WMEs for each group, with data averaged over blocks of six trials. A two-way ANOVA was computed based on the scores. The analysis revealed a significant main effect of blocks of trials ( $\mathrm{F}=15.97$; $\mathrm{P}<0.0001)$ and groups (Control and C. longa extract-fed group) ( $\mathrm{F}=122.3 ; \mathrm{P}<0.0001)$ on the number of WMEs. The analysis also revealed a significant block and group interaction $(\mathrm{F}=3.385 ; \mathrm{P}=0.0014)$.

\subsection{Effect of Oral Administration of $C$. longa Extract on the Levels of Lipid Peroxide (LPO) in the Cortico-Hippocampal Brain Tissues of the Old Rats}

Aging is characterized by a progressive decline in the efficiency of tackling the oxidative stress. The impaired functional capacity of many systems characterizes aging. When such impairments occur in the brain, the susceptibility to neurodegenerative diseases amplifies considerably. The free radical theory of aging posits that the functional impairments in brains are due to the attack on critical cellular components by free radicals and reactive oxygen species produced during normal metabolism. Therefore, the effect of chronic oral administration of C. longa extract on the oxidative stress of the brain cortex/hippocampal tissues was evaluated. Chronic oral administration of $C$. longa extract significantly decreased the levels of LPO in the cortico-hippocampal brain tissues, as compared to those of the control rats (Figure 2). The results thus suggest that free radicals, at least partially, responsible for the functional impairment in aged brains, could be ameliorated by the $C$. longa extract.

\subsection{Effect of Oral Administration of C. longa Extract on the Levels of TNF- $\alpha$ in the Brain Cortex and Hippocampus of the Old Rats}

Tumor necrosis factor alpha (TNF- $\alpha$ ) is a cytokine produced mainly by cells of the immune system. It is also expressed by brain neurons and glia and seen associated with neurodegenerative diseases of the brain. Therefore, the effects of the oral administration of $C$. longa extract on the levels of the TNF $\alpha$ in the cortex/hippocampus tissues were examined. Chronic oral administration of $C$. longa extract significantly decreased the levels of TNF- $\alpha$ both in the cortex ( 25\%) and hippocampus ( 18\%), as compared to those of the control rats (Figure 3).

\subsection{Effects of Preadministration of $C$. longa Extract on the Levels of LPO in the Brains of Hypoxic Rats}

We report a significant increase of the oxidative stress in the hypoxia-lesioned rats, as demonstrated by the significant increase of the LPO levels in both the cortex and hippocampal tissues, when compared to those of the nonhypoxic control rats. The hypoxic rats pretreated with C. longa extract (Hypoxia + CLE rats) had significantly decreased levels of LPO both in the cortex and hippocampal tissues, as compared to those of the hypoxic rats (Figure 4). The extract-alone also significantly reduced the levels of LPO in the cortex or hippocampus, as compared to those in the control rats. Finally, these results demonstrate a significant inhibition of oxidative stress instigated by the hypoxia-reperfusion injury in the brains of the old rats. 


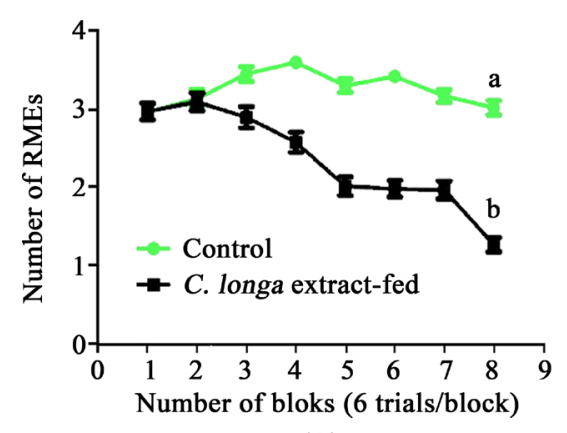

(A)

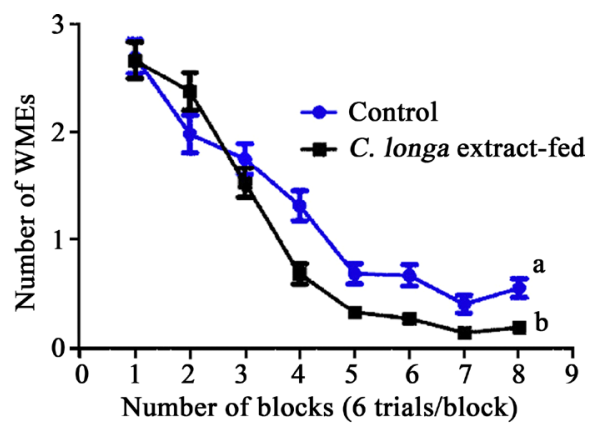

(B)

Figure 1. Effects of oral administration of $C$. longa extract on reference memory errors (RMEs) (A) and working memory errors (WMEs) (B). Data are the mean \pm SEM for each block of six trials. Data were analyzed by randomized block two-way (block and group) ANOVA.
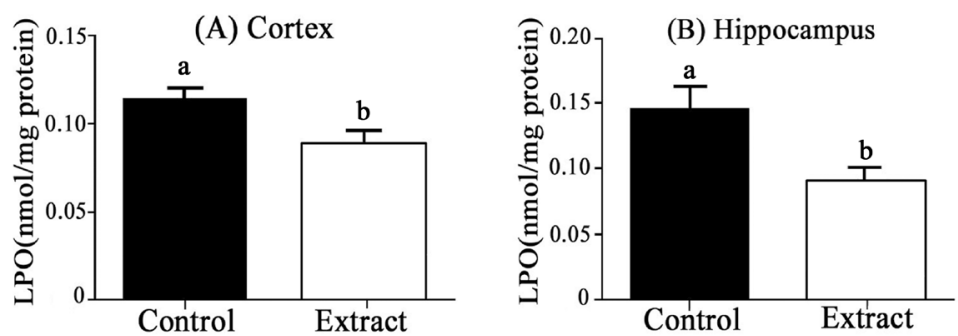

Figure 2. Effect of oral administration of C. longa on the levels of lipid peroxide (LPO) of cortex (A) and hippocampus (B) of old rats. Each bar $=$ mean \pm SEM,$n=6-7$. Bars with different alphabets are significantly different at $P<0.05$. Data were analyzed by unpaired student's $t$-test.

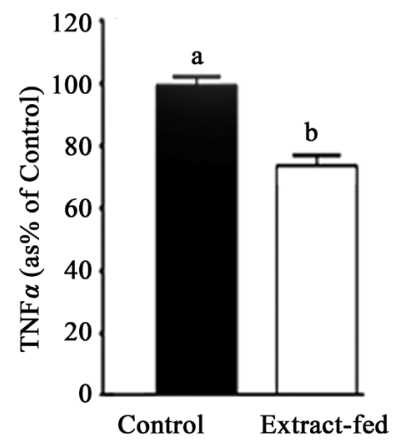

(A)

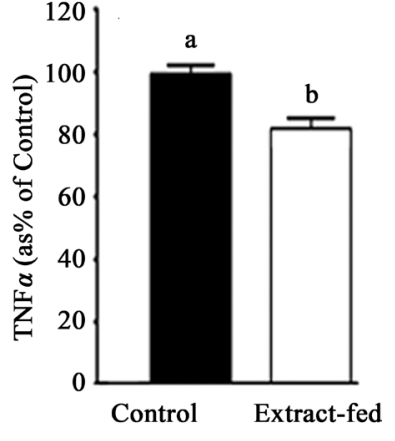

(B)

Figure 3. Effect of oral administration of C. longa on the levels of TNF- $\alpha$ of the cortex (A) and hippocampus (B) of aged rats. Bars with different alphabets are significantly different at $\mathrm{P}<0.05$ (unpaired student's $t$-test).
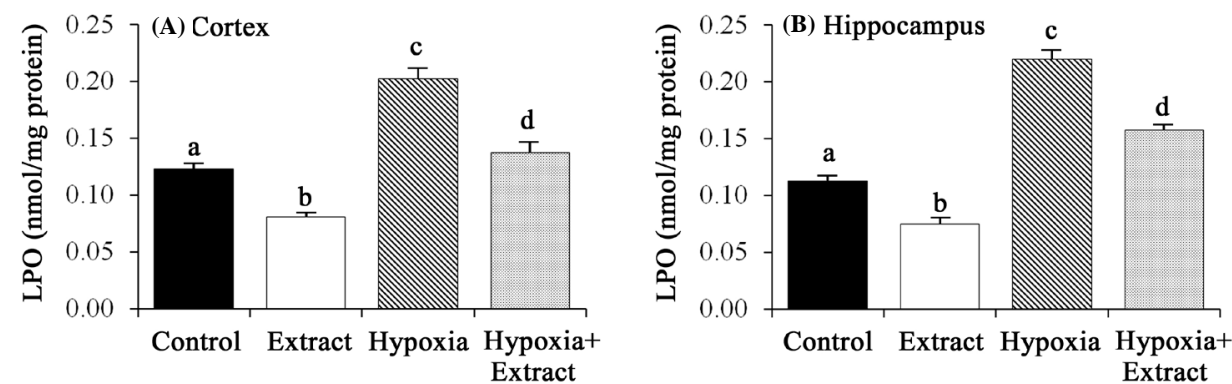

Figure 4. Effect of preadminstration of C. longa extract on the levels of LPO of the cortex (A) and hippocampus (B) tissues of the hypoxic rats. Each bar represents mean \pm SEM, $n=6$. Bars with different alphabets are significantly different at $P<$ 0.05 (one-way ANOVA). 


\subsection{Histological Analysis of the Hippocampus of the Hypoxic Rats}

Hippocampus is considered as the key structure for memory formation, thus histology of the hippocampus is reported in this investigation. Figure 5 shows the histological appearance of hippocampus in Control, Extract-administrated only, Hypoxia-only, and Hypoxia + Extract-preadministrated of rats. A distinguished perfect cell arrangement was found in histological examinations of the brain hippocampus slice of $C$. longa extract-fed rats (Figure 5(B)) than that of normal control rats (Figure 5(A)) brain slice. Pyramidal cell layers contained more healthy and perfect cells in extract-fed rat brain slice (Figure 5(B)). Characteristic cell swelling and broken cell membranes were found in the hypoxic rat brain in contrast with those in the non-hypoxic controls and extract-fed hypoxic rat brain. In the hippocampus of hypoxic rats, structures of the CA region were more dented; pyramidal cell layer contained more inflammatory cells and was disseminated (Figure 5(C)), whereas more normal and less inflamed pyramidal cell layer was found in the hippocampus of extract-fed hypoxic rat brains (Figure 5(D)).

\subsection{In Vitro Antioxidative Properties of C. longa Extract}

Antioxidative phytoconstituents, including total polyphenols and flavonoid contents were determined in triplicate. Total polyphenol contents of the $C$. longa extract were $46.8 \pm 1.5 \mathrm{mg} / \mathrm{g}$ of the extract against gallic acid equivalent. Total flavonoid contents were $180 \pm 6.8 \mathrm{mg} / \mathrm{g}$ of the extract, against quercetin equivalent. After 10 min of co-incubation with DPPH $(0.2 \mathrm{mM})$, the percentage scavenging activity of curcumin standard $(0-400$ $\mathrm{mM}$ ) ranged $38 \%-71 \%$, while that of the extract of the C. longa $(0-1.0 \mathrm{mg} / \mathrm{ml})$ ranged $34 \%-52 \%$, thus indicating a comparable free radical scavenging activity of the $C$. longa extract (Figure 6(A)). The rate of the scanvanging activity was also comparable with that of the pure curcumin standard, thus again suggesting the presence of a considerable amount of curcumin compound in the crude extract of the $C$. longa. The DPPH scavenging rates of $C$. longa $(125 \mu \mathrm{g} / \mathrm{ml}$ extract) were $14 \%$ - 20\% lower than those of the pure curcumin standard compound $(50 \mu \mathrm{M})$ (Figure 6(B)).

The anti-LPO activity of the C. longa extract was evaluated by its ability to inhibit in vitro Fenton's reagent-induced oxidative stress (OS) in the fresh hippocampal tissue homogenates. Fenton's reagent significantly increased the oxidative stress in the brain tissues (Figure 7), as evident by the increased levels of LPO. However, the co-treatment of the Fenton's reagent with $C$. longa extract significantly decreased the oxidative stress (Figure 7). The results thus demonstrate a strong anti-LPO effect of $C$. longa extract.
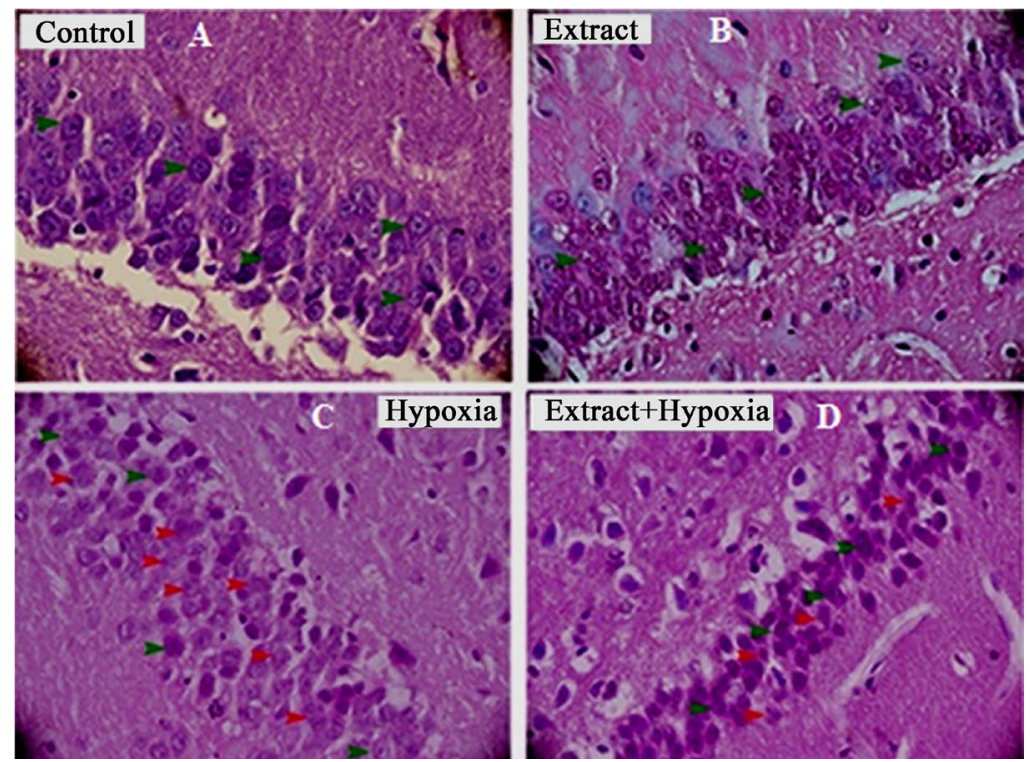

Figure 5. Hematoxylene and eosin dye stained representative slices of brain hippocampus. (A) Control rats; (B) C. longaalone fed rats; (C) Hypoxic alone rats; (D) C. longa-pretreated + hypoxic rats. Here, green arrows show normal cell with normal anatomical characteristics, clear nuclei, and red arrows showed cell swelling, cell rupture, diffused cell membranes and nuclei. Picture at $100 \times$ magnification. 

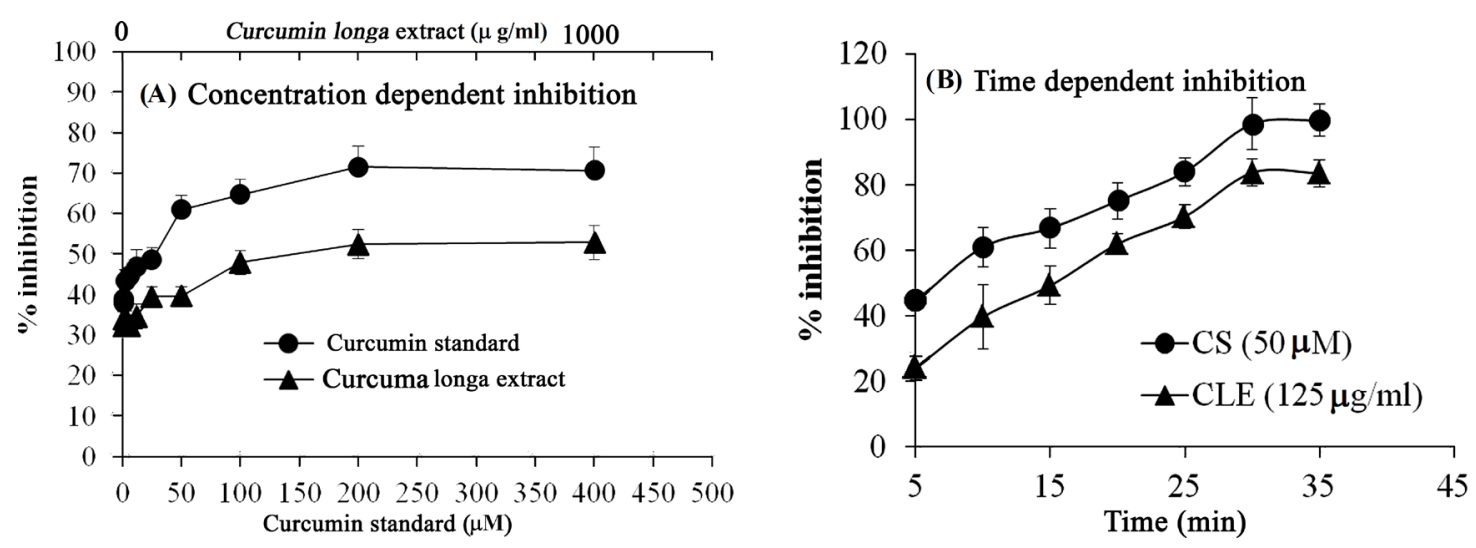

Figure 6. The DPPH free radical scavenging activity of C. longa extract and standard pure curcumin. (A) Concentration-dependent scavenging of DPPH-free radical; (B) Rate of DPPH-free scavenging at a given concentration of pure curcu$\min (\mathrm{CS}, 50 \mu \mathrm{M})$ and $C$. longa extract $(125 \mu \mathrm{g} / \mathrm{ml})$. Each data points indicate mean of three independent experiments.

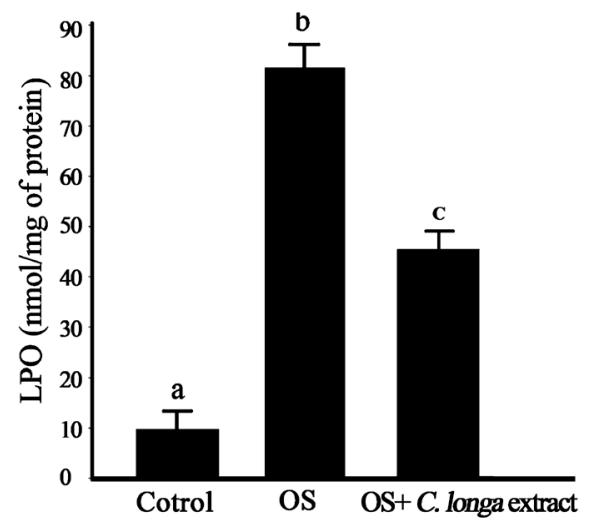

Figure 7. The effect of in vitro co-treatment $C$. longa extract on Fenton's reagent-induced oxidative stress (OS) in the rat hippocampus homogenate. The bars represent the mean \pm SEM (Standard error of the mean; $n=3$ ). Control = homogenate-only; OS = Fenton's reagent-induced oxidative stress in the homogenates; OS $+C$. longa extract = Fenton's reagent-induced oxidative stress $+C$. longa extract in the homogenates. Data were analyzed by one-way ANOVA followed by Tukey's least square differences test for post hoc comparisons. Bars with different alphabets are significantly different at $\mathrm{P}<$ 0.05 .

\section{Discussion}

The results of the present investigation clearly demonstrate that the chronic oral administration of $C$. longa extract improves spatial memory-related learning ability in old rats. The oral administration of $C$. longa extract concomitantly ameliorated the levels of proinflammatory tumor necrosis factor-alpha (TNF- $\alpha$ ) and oxidative stress in the cortico-hippocampal brain tissues, as indicated by the decreased levels of lipid peroxide (LPO) and TNF- $\alpha$ in the C. longa extract-fed old rats. The extract-treatment did not have an influence on the body weight of the rats and affect the liver/kidney functions-related enzymes, including glutamate-pyruvate transaminase, glutamate-oxaloacetate transaminase, gama-glutamyltransferase and creatine kinase, indicating that the $C$. longa extract (at $100 \mathrm{mg} / \mathrm{kg}$ BW/day) did not exert detrimental effects on the rats (data not shown).

Eight-armed radial maze behavior allows the measurement of spatial (navigation) memory (long and shortterm) in terms of reference memory and working memory errors without any harmful effects on the rats [16]. It is also the most popular protocol to study the spatial memory performance. We have also previously used this protocol to evaluate the navigation-related spatial memory of old and Alzheimer's disease model rats, and there was a significant positive correlation between the cortico-hippocampal levels of LPO and long-term memory (RMEs score) [24]. The oral administration of C. longa extract significantly decreased the numbers of RMEs/ WMEs scores, thus suggesting an improvement of spatial learning-related long-term and short-term memory, respectively, in the extract-fed rats (Figure 1(A), Figure 1(B)). The oxidative stress, which is associated with 
aging and neurodegenerative diseases such as Alzheimer's disease, leads to a decline in spatial memory-related learning ability [28] [29]. Thus, the result of the decreased levels of LPO in the cortex and hippocampus of the extract-fed rats might be related to the ameliorative effect of $C$. longa extract on the oxidative stress. Gamoh et al. [30], Goto et al. [31] also reported that the impairment of cognitive performances in older rats is related with increases in the oxidative stress. As hippocampus is considered as the key structure of memory formation, increased levels of LPO, reference memory/working memory error scores are positively correlated [16]. Interruption of hippocampal pathways causes crucial memory deficits in the radial-arm maze task [32]. C. longa extract exhibited DPPH-free radical scavenging activity and inhibited in vitro Fenton's-reagent-induced oxidative stress in the rat brain hippocampal tissues. Curcumin from turmeric has been shown to ameliorate oxidative stress and it is considered to be a potent antioxidant in hepatic tissues [33], endothelial cells [34] and neuroprotective effects [35]. We thus speculate that the improvement of spatial learning ability in the $C$. longa extract-fed rats, as compared to that of the controls, was, at least partially, due to the ameliorative effect of $C$. longa extract on the oxidative stress. The speculation is consistent with the presence of substantial amount of total polyphenols and flavonoid contents in the $C$. longa extract. The assumption is further strengthened by the DPPH-free radical scavenging activities and in vitro anti-LPO effect of the extract (Figure 6). The results of our investigation are also consistent with the reports of Pyrzanowska et al. (2010) [14], where learning ability after C. longa extract treatment was enhanced in Morris water maze.

TNF- $\alpha$ is prominent among proinflammatory cytokines known to be associated with neuropathological effects underlying several neurodegenerative disorders, including Alzheimer's disease [36] [37]. TNF- $\alpha$ mediates synaptic loss and memory impairments [38]. More recently, Liu et al. (2013) reported that TNF $\alpha$-deficient mice display better spatial memories [39]. These reports thus suggest that the act of suppression of TNF- $\alpha$ is helpful in learning and improving the memory functions. Oxidative stress mediates TNF- $\alpha$-induced cellular damage [40]. Chronic administration of $C$. longa extract significantly decreased the levels of TNF- $\alpha$, concurrently with decreases in the levels of oxidative stress in the hippocampal tissues, thus again demonstrating that the $C$. lon$g a$-induced decreases in the levels of TNF- $\alpha$, is, at least partially, associated with ameliorative effects of $C$. lon$g a$ on the spatial memory of the old rats.

To corroborate the antioxidative effects (results) of $C$. longa extract on the brains of the rats of the maze-tests, we directly induced oxidative stress in the brains of $C$. longa-pretreated rats by inducing hypoxia-reperfusion injury, as described previously [21]. Occlusion of the common carotid arteries causes cerebral blood-hypoperfusion which ultimately leads the cell to undergo apoptosis as a response to excessive free radical generation [41]. Again, hippocampus is crucially involved in spatial memory while the cortex is necessary for working memory and executive function [42]. The hippocampus slices of the hypoxic rats displayed swelling characteristics of cells, and cell membranes of the hippocampus in these rats were found broken in contrast with those of the non-hypoxic control hippocampal slices. In the Hypoxia $+C$. longa extract-fed rats, the pyramidal cell layer of the hippocampus was visually normal and there was much less inflammation, as compared to that of the control rats. Therefore, these results suggest that carotid artery occlusion conferred oxidative stress to the cells and resulted in brain cell damage in the hippocampus. Moreover, the hypoxia-induced oxidative stress was evident biochemically, as indicated by the in vivo increased levels of LPO in the hippocampal homogenates (Figure 4(B)). However, histology of the C. longa extract-pretreated hypoxic (Hypoxia + Extract) rats exhibited less-swelling characteristics and/or cells with very few damages. Most of the cells in the Hypoxia + C. longa extract-fed rats retained normal cellular structure, thus demonstrating an extract-mediated protective effect against cellular damages in the hippocampal brain tissues. The activities of the hepatorenal functions-related serum enzymes were not altered in either of the rat groups, thus suggesting that the $C$. longa extract did not have any detrimental effect(s) on liver and kidneys (data not shown) of the experimental rats. Rather, the oral administration of $C$. longa extract had beneficial effects on the proinflammatory $\mathrm{TNF} \alpha$, oxidative stress and degenerative changes in the normal and hypoxic aged rats. Again, the ameliorative effect is supported by the presence of substantial amounts of antioxidants such as total polyphenol/flavonoids, and direct evidence of the in vitro anti-LPO activities.

The oral administration of $C$. longa significantly ameliorated both the short-term and long-term memory capacities of the aged rats. Short-term memory, which only lasts for a short period of time, does not require any protein synthesis or remodeling of the synapse, while the long-term memory formation requires de novo protein synthesis [43]. It is believed that short-term memory is a primary function of the prefrontal cortex. Once memory is consolidated, it is carried over to the hippocampus wherein long-term memories are formed and stored 
permanently. This conversion from short-term to long-term memory requires concerted effort, the passage of time, and the absence of interference in memory consolidation. Besides its role as antioxidant and anti-inflammation, the beneficial effects of $C$. longa extract might also have evolved from other effects on the complex mechanism(s) of memory.

\section{Conclusion}

Hippocampus is considered as the key structure for the memory formation [44]. Furthermore, impairment of cognitive performance in older rats relates with increases of oxidative stress [30] [31]. It is thus speculated that the improvement of spatial memory-related learning ability of aged rats was due to the ameliorating effect of extract on the oxidative stress and proinflammatory TNF $\alpha$. Finally, the results of this investigation revealed that extract of $C$. longa provides beneficial effects on the learning ability of old rats by providing a potential antioxidative and anti-inflammatory defense and that curcumin pre-treatment may lead to increased protection against cognitive decline associated with aging and neurodegenerative diseases, such as Alzheimer's disease. However, further investigations are indeed necessary for the exact mechanism of actions of $C$. longa extract.

\section{Acknowledgements}

Authors specially acknowledge to University Grant Commission-World Bank’s HEQEP project (CP358) for providing the instrumental supports of this work.

\section{Conflict of Interests}

The authors declare that there is no conflict of interests regarding the publication of this paper.

\section{References}

[1] Goel, A., Kunnumakkara, A.B. and Aggarwal, B.B. (2007) Curcumin as Curecumin: from Kitchen to Clinic. Biochemical Pharmacology, 75, 787-809. http://dx.doi.org/10.1016/j.bcp.2007.08.016

[2] Aggarwal, B.B. and Harikumar, K.B. (2009) Potential Therapeutic Effects of Curcumin, the Anti-Inflammatory Agent against Neurodegenerative, Cardiovascular, Pulmonary, Metabolic, Autoimmune and Neoplastic Diseases. International Journal of Biochemistry \& Cell Biology, 41, 40-59. http://dx.doi.org/10.1016/j.biocel.2008.06.010

[3] Hatcher, H., Planalp, R. Cho, J., Torti, F.M. and Torti, S.V. (2008) Curcumin: From Ancient Medicine to Current Clinical Trials. Cellular and Molecular Life Sciences, 65, 1631-1652. http://dx.doi.org/10.1007/s00018-008-7452-4

[4] Hiserodt, R., Hartman, T.G., Ho, C.T. and Rosen, R.T. (1996) Characterization of Powdered Turmeric by Liquid Chromatography-Mass Spectrometry and Gas Chromatography-Mass Spectrometry. Journal of Chromatography, 740, 51-63. http://dx.doi.org/10.1016/0021-9673(96)00103-3

[5] Bala, K., Tripathy, B.C. and Sharma, D. (2006) Neuroprotective and Antiageing Effects of Curcumin in Aged Rat Brain Regions. Biogerontology, 7, 81-89. http://dx.doi.org/10.1007/s10522-006-6495-X

[6] Ramirez-Tortosa, M.C., Mesa, M.D., Aguilera, M.C., Quiles, J.L., Baro, L., Ramirez-Tortosa, C.L., Martinez-Victoria, E. and Gil, A. (1999) Oral Administration of Turmeric Extract Inhibits LDL Oxidation and Has Hypocholesterolemic Effects in Rabbits with Experimental Atherosclerosis. Atherosclerosis, 147, 371-378. http://dx.doi.org/10.1016/S0021-9150(99)00207-5

[7] Ono, K., Hasegawa, K., Naiki, H. and Yamada, M. (2004) Curcumin Has Potent Anti-Myloidogenic Effects for Alzheimer's Beta-Amyloid Fibrils in Vitro. Journal of Neuroscience Research, 75, 742-750. http://dx.doi.org/10.1002/jnr.20025

[8] Yang, F., Lim, G.P., Begum, A.N., Ubeda, O.J., Simmons, M.R., Ambegaokar, S.S., Chen, P.P., Kayed, R., Glabe, C.G., Frautschy, S.A. and Cole, G.M. (2005) Curcumin Inhibits Formation of Amyloid Beta Oligomers and Fibrils, Binds Plaques and Reduces Amyloid in Vivo. Journal of Biological Chemistry, 280, 5892-5901. http://dx.doi.org/10.1074/jbc.M404751200

[9] Garcia-Alloza, M., Borrelli, L.A., Rozkalne, A., Hyman, B.T. and Bacskai, B.J. (2007) Curcumin Labels Amyloid Pathology in Vivo, Disrupts Existing Plaques and Partially Restores Distorted Neurites in an Alzheimer Mouse Model. Journal of Neurochemistry, 102, 1095-1104. http://dx.doi.org/10.1111/j.1471-4159.2007.04613.x

[10] Frautschy, S.A., Hu, W., Kim, P., Miller, S.A., Chu, T., Harris-White, M.E. and Cole, G.M. (2001) Phenolic Anti-Inflammatory Antioxidant Reversal of Abeta-Induced Cognitive Deficits and Neuropathology. Neurobiology of Aging, 22, 993-1005. http://dx.doi.org/10.1016/S0197-4580(01)00300-1 
[11] Kim, H.Y., Park, E.J., Joe, E.H. and Jou, I. (2003) Curcumin Suppresses Janus Kinase-STAT Inflammatory Signaling through Activation of Src Homology 2 Domain-Containing Tyrosine Phosphatase 2 in Brain Microglia. The Journal of Immunology, 171, 6072-6079. http://dx.doi.org/10.4049/jimmunol.171.11.6072

[12] Jung, K.K., Lee, H.S., Cho, J.Y., Shin, W.C., Rhee, M.H., Kim, T.G., Kang, J.H., Kim, S.H., Hong, S. and Kang, S.Y. (2006) Inhibitory Effect of Curcumin on Nitric Oxide Production from Lipopolysaccharide-Activated Primary Microglia. Life Sciences, 79, 2022-2031. http://dx.doi.org/10.1016/j.lfs.2006.06.048

[13] Zhao, B.L.X., Li, J., He, R.G., Cheng, S.J. and Xin, W.J. (1989) Scavenging Effect of Extracts of Green Tea and Natural Antioxidants on Active Oxygen Radicals. Cell Biophysics, 14, 175-185. http://dx.doi.org/10.1007/BF02797132

[14] Pyrzanowska, J., Piechal, A., Klin, K.B., Lehner, M., Skórzewska, A., Turzyńska, D., Sobolewska, A., Plaznik, A. and Tyszkiewicz, E.W. (2010) The Influence of the Long-Term Administration of Curcuma longa Extract on Learning and Spatial Memory as Well as the Concentration of Brain Neurotransmitters and Level of Plasma Corticosterone in Aged Rats. Pharmacology Biochemistry and Behavior, 95, 351-358. http://dx.doi.org/10.1016/j.pbb.2010.02.013

[15] Hodges, H. (1996) Maze Procedures: The Radial-Arm and Water Maze Compared. Cognitive Brain Research, 3, 167181. http://dx.doi.org/10.1016/0926-6410(96)00004-3

[16] Gamoh, S., Hashimoto, M., Sugioka, K., Hossain, S.M., Hata, N., Misawa, Y. and Masumura, S. (1999) Chronic Administration of Docosahexaenoic Acid Improves Reference Memory Related Learning Ability in Young Rats. Neuroscience, 93, 237-241. http://dx.doi.org/10.1016/S0306-4522(99)00107-4

[17] Gerrard, J.L., Burke, S.N., McNaughton, B.L. and Barnes, C.A. (2008) Sequence Reactivation in the Hippocampus Is Impaired in Aged Rats. The Journal of Neuroscience, 28, 7883-7890. http://dx.doi.org/10.1523/JNEUROSCI.1265-08.2008

[18] Ohkawa, H., Ohnishi, N. and Yagi, K. (1979) Assay for Lipid Peroxidation in Animal Tissue by Thiobarbituric Acid Reaction. Analytical Biochemistry, 95, 351-358. http://dx.doi.org/10.1016/0003-2697(79)90738-3

[19] Haque, M., Islam, J., Rahaman, A., Selina, F.A., Rahman, M.A., Hasan, M. and Hossain, S. (2014) Raphanus sativus Ameliorates Atherogeneic Lipid Profiles in Hypercholesterolemic Rats and Hypercholesterolemia-Associated Peroxidative Liver Damage. Journal of Advanced Chemistry, 7, 1385-1394.

[20] Lowry, O.H., Rosebrough, N.J., Farr, A.L. and Randall, R.J. (1951) Protein Measurement with the Folin Reagent. The Journal of Biological Chemistry, 193, 265-275.

[21] Islam, J., Haque, M., Rahaman, A. and Hossain, S. (2014) Syzygium cumini (L.) Seed Extract Protects Embryofoetal Brains against Intrauterine Oxidative Toxicity in Rats during Hypoxia-Reperfusion Injury. International Journal of Pharmaceutical Sciences and Research, 3, 170-177.

[22] Iwasaki, Y., Ito, S., Suzuki, M., Nagahori, T., Yamamoto, T. and Konno, H. (1989) Forebrain Ischemia Induced by Temporary Bilateral Common Carotid Occlusion in Normotensive Rats. Journal of the Neurological Sciences, 90, 155165. http://dx.doi.org/10.1016/0022-510X(89)90098-1

[23] Raghavendra, M., Trigunayat, A., Singh, R.K., Mitra, S., Goel, R.K. and Acharya, S.B. (2007) Effect of Ethanolic Extract of Root of Pongamia pinnata (L.) Pierre on Oxidative Stress, Behavioral and Histopathological Alterations Induced by Cerebral Ischemia-Reperfusion and Long-Term Hypoperfusion in Rats. Indian Journal of Experimental Biology, 45, 868-876.

[24] Rahaman, A., Hossain, S., Rahman, M., Hossain, I., Nahar, T., Uddin, B. and Khalil, I. (2013) Syzygium cumini (L.) Seed Extract Improves Memory Related Learning Ability of Old Rats in Eight Arm Radial Maze. Journal of Pharmacognosy and Phytochemistry, 1, 85-94.

[25] Amin, I., Norazaidah, Y. and Hainida, K.I.E. (2006) Antioxidant Activity and Phenolic Content of Raw and Blanched Amaranthus Species. Food Chemistry, 94, 47-52. http://dx.doi.org/10.1016/j.foodchem.2004.10.048

[26] Kumar, S., Kumar, D., Manjusha, K., Saroha, S.N. and Vashishta, B. (2008) Antioxidant Free Radical Scavenging Potential of Citrullus colocynthis (L.) Schrad. Methanolic Fruit Extract. Acta Pharmaceutica, 58, 215-220.

[27] Hossain, S., Rahaman, A., Nahar, T., Basunia, M.A., Rahman, F., Uddin, B., Shahriar, M. and Mahmud, I. (2011) Syzygium cumini (L.) Skeels Seed Extract Ameliorates in Vitro and in Vivo Oxidative Potentials of the Brain Cerebral Cortex of Alcohol-Treated Rats. Oriental Pharmacy and Experimental Medicine, 12, 59-66. http://dx.doi.org/10.1007/s13596-011-0044-0

[28] Barnes, C.A., Nadel, L. and Honig, W.K. (1980) Spatial Memory Deficit in Senescent Rats. Canadian Journal of Psychology, 34, 29-39. http://dx.doi.org/10.1037/h0081022

[29] Gallagher, M. and Burwell, R.D. (1989) Relationship of Age-Related Decline across Several Behavioral Domains. Neurobiology of Aging, 10, 691-708. http://dx.doi.org/10.1016/0197-4580(89)90006-7

[30] Oler, J.A. and Markus, E.J. (1998) Age-Related Deficits on the Radial Maze and in Fear Conditioning: Hippocampal Processing and Consolidation. Hippocampus, 8, 402-415. http://dx.doi.org/10.1002/(SICI)1098-1063(1998)8:4<402::AID-HIPO8>3.0.CO;2-I 
[31] Goto, S., Naito, H., Kaneko, T., Chung, H.Y. and Radak, Z. (2007) Hormetic Effects of Exercise in Aging: Correlation with Oxidative Stress. Applied Physiology, Nutrition, and Metabolism, 32, 948-953. http://dx.doi.org/10.1139/H07-092

[32] Jerrard, L.E. and Okaichi, H. (1984) On the Role of Hippocampal Connections in the Performance of Place and Clue Tasks: Comparisons with Damage to Hippocampus. Behavioral Neuroscience, 98, 946-954. http://dx.doi.org/10.1037/0735-7044.98.6.946

[33] Eybl, V., Kotyzova, D. and Koutensky, J. (2006) Comparative Study of Natural Antioxidants-Curcumin, Resveratrol and Melatonin-In Cadmium-Induced Oxidative Damage in Mice. Toxicology, 225, 150-156. http://dx.doi.org/10.1016/j.tox.2006.05.011

[34] Motterlini, R., Foresti, R., Bassi, R. and Green, C.J. (2000) Curcumin, an Antioxidant and Anti-Inflammatory Agent, Induces Heme Oxygenase-1 and Protects Endothelial Cells against Oxidative Stress. Free Radical Biology \& Medicine, 28, 1303-1312. http://dx.doi.org/10.1016/S0891-5849(00)00294-X

[35] Rajakrishnan, V., Viswanathan, P., Rajasekharan, K.N. and Menon, V.P. (1999) Neuroprotective Role of Curcumin from Curcuma longa on Ethanol-Induced Brain Damage. Phytotherapy Research, 13, 571-574. http://dx.doi.org/10.1002/(SICI)1099-1573(199911)13:7<571::AID-PTR494>3.0.CO;2-7

[36] Fillit, H., Ding, W.H., Buee, L., Kalman, J., Altstiel, L., Lawlor, B. and Wolf-Klein, G. (19991) Elevated Circulating Tumor Necrosis Factor Levels in Alzheimer's Disease. Neuroscience Letters, 129, 318-320. http://dx.doi.org/10.1016/0304-3940(91)90490-K

[37] Boka, G., Anglade, P., Wallach, D., Javoy-Agid, F., Agid, Y. and Hirsch, E.C. (1994) Immunocytochemical Analysis of Tumor Necrosis Factor and Its Receptors in Parkinson's Disease. Neuroscience Letters, 172, 151-154. http://dx.doi.org/10.1016/0304-3940(94)90684-X

[38] Lourenco, M.V., Clarke, J.R., Frozza, R.L., Bomfim, T.R., Forny-Germano, L., Batista, A.F., et al. (2013) TNF- $\alpha$ Mediates PKR-Dependent Memory Impairment and Brain IRS-1 Inhibition Induced by Alzheimer's $\beta$-Amyloid Oligomers in Mice and Monkeys. Cell Metabolism, 18, 831-843. http://dx.doi.org/10.1016/j.cmet.2013.11.002

[39] Liu, B., Zupan, B., Laird, E., Klein, S., Gleason, G., Bozinoski, M., Toth, J.G. and Toth, M. (2013) Maternal Hematopoietic TNF, via Milk Chemokines, Programs Hippocampal Development and Memory. Nature Neuroscience, 17, 97105. http://dx.doi.org/10.1038/nn.3596

[40] Suematsu, N., Tsutsui, H., Wen, J., Kang, D., Ikeuchi, M., Ide, T., Hayashidani, S., Shiomi, T., Kubota, T., Hamasaki, N. and Takeshita, A. (2003) Oxidative Stress Mediates Tumor Necrosis Factor-Alpha-Induced Mitochondrial DNA Damage and Dysfunction in Cardiac Myocytes. Circulation, 107, 1418-1423. http://dx.doi.org/10.1161/01.CIR.0000055318.09997.1F

[41] Schaller, B. and Graf, R. (2004) Cerebral Ischemia and Reperfusion: The Pathophysiologic Concept as a Basis for Clinical Therapy. Journal of Cerebral Blood Flow \& Metabolism, 24, 351-371. http://dx.doi.org/10.1097/00004647-200404000-00001

[42] Fischer, W., Gage, F.H. and Björklund, A. (1989) Degenerative Changes in Forebrain Cholinergic Nuclei Correlate with Cognitive Impairments in Aged Rats. European Journal of Neuroscience, 1, 34-45. http://dx.doi.org/10.1111/j.1460-9568.1989.tb00772.x

[43] Davis, H.P. and Squire, L.R. (1984) Protein Synthesis and Memory. Psychological Bulletin, 96, 518-559. http://dx.doi.org/10.1037/0033-2909.96.3.518

[44] Burke, S.N. and Barnes, C.A. (2006) Neural Plasticity in the Ageing Brain. Neuroscience, 7, 30-40. http://dx.doi.org/10.1038/nrn1809 